\title{
Tecnura
}

\section{Productividad en una celda de manufactura flexible simulada en promodel utilizando path networks type crane}

\author{
Productivity in a flexible manufacturing cell simulated \\ in promodel using path networks type crane
}

\begin{abstract}
María Elena Bernal Loaiza*, German Cock Sarmiento**, Jorge Hernán Restrepo Correa***
\end{abstract}
Fecha de recepción: 31 de agosto de 2013

Fecha de aceptación: 5 de diciembre de 2014

Citation / Para citar este artículo: Bernal Loaiza, M. E., Cock Sarmiento, G., \& Restrepo Correa, J. H. (2015). Productividad en una celda de manufactura flexible simulada en promodel utilizando path networks type crane. Revista Tecnura, 19(44), 133-144. doi:http://dx.doi.org/10.14483/udistrital.jour.tecnura.2015.2.a10

\section{Resumen}

Esta investigación se centra en el diseño de una simulación del proceso de una celda de manufactura flexible (FMC) de la Facultad de Ingeniería Industrial de la Universidad Tecnológica de Pereira, con el propósito de medir y elegir aquella alternativa de solución que mejore en mayor grado la productividad del sistema actual utilizando los resultados que proporciona el software Promodel. Para realizar lo anterior se utilizaron etapas de la simulación como definición del sistema, formulación del modelo, identificación de variables, recolección de datos, implementación e interpretación.

Como resultado, se calculó la productividad teniendo en cuenta las variables del modelo y tiempo de la simulación, al mismo tiempo se interpretó la información de cada una de las locaciones, hallando el porcentaje de utilización y total de entidades para las locaciones con capacidad unitaria; para las locaciones con mayor capacidad se encuentra información referente al porcentaje de tiempo vacío, parcialmente ocupado, lleno y no disponible respecto del tiempo disponible. A diferencia de otras simulaciones, en este diseño en la parte de redes se utilizó el tipo de red Crane, asociado a los recursos del modelo. El cual facilita el desplazamiento de los brazos robots con las piezas que circulan a través del sistema.

Palabras clave: fabricación asistida por ordenador, modelo de simulación, productividad.

\begin{abstract}
This research focuses on the design of a simulation of the process in a flexible manufacturing cell at the Faculty of Engineering of the Universidad Tecnológica de Pereira, aiming at measuring and choosing the one alternative of solution that improves to a greater degree the productivity of the current system using the results provided by the software Promodel. In order to do this, the stages of the simulation as definition of the system, model formulation, identification
\end{abstract}

* Ingeniera de Sistemas, magíster en Investigación de Operaciones y Estadística, candidata a magíster en Administración del desarrollo Humano y Organizacional. Docente de la Universidad Tecnológica de Pereira, Pereira, Colombia. Contacto: mbernal@utp.edu.co

** Ingeniero Industrial, magíster en Investigación de Operaciones y Estadística. Docente de la Universidad Tecnológica de Pereira, Pereira, Colombia. Contacto: cook20038@gmail.com

*** Ingeniero Industrial, magíster en Investigación de Operaciones y Estadística, énfasis en Producción. Docente de la Universidad Tecnológica de Pereira; director, maestría y especialización en Sistemas Integrados de Gestión de la Calidad. Pereira, Colombia. Contacto: jhrestrepoco@utp.edu.co 
of variables, data collection, implementation and interpretation were used.

As a result, the productivity was calculated taking into account the variables of the model and simulation time, at the same time the information from each of the locations was interpreted, finding the percentage of use and total number of entities for locations with unit capacity, and for locations with greater capacity there is information concerning to the percentage of empty time, partially occupied, full and unavailable regarding available time. In contrast to other simulations, in this design, on the networks the type of network Crane was used, associated to the resources of model. Which facilitates the movement of robot arms with the parts circulating through the system.

Keywords: computer aided manufacturing, productivity, simulation models.

\section{INTRODUCCIÓN}

La productividad en una celda de manufactura flexible Flexible Manufacturing Cells (FMC) puede medirse en cuanto al mejoramiento en calidad, la reducción en costos e inventario, y un mejor manejo de los productos.

Una FMC es un grupo de máquinas o estaciones de trabajo relacionadas que realizan una tarea específica. El proceso que ejecuta cada una de las estaciones de trabajo de una FMC pueden ser simuladas en Promodel, con el fin de predecir posibles mejoras para el aumento de la productividad. Este aumento de productividad está enmarcado en los esfuerzos que conlleva una toma de decisión para un mejoramiento de la calidad de los procesos.

Por otra parte, la simulación ha demostrado ser capaz de hacer frente a las tareas de mejora de la productividad y la eficiencia en las que estas dificultades se superponen e interactúan. Históricamente, la mayor parte del éxito de la simulación que ha tenido en otros sectores de la economía (por ejemplo, el servicio, el transporte y el cuidado de la salud) se ha debido en gran medida a la reputación que se ganó en el sector manufacturero (Kale, 2007).

Teniendo en cuenta lo anterior, el presente trabajo tiene como objetivo realizar una simulación de la celda de manufactura flexible de la Facultad de Ingeniería Industrial de la Universidad Tecnológica de Pereira, utilizando el software ProModel, donde se describe cómo se utiliza la simulación para analizar la productividad mediante la construcción de un modelo del sistema actual de la celda, haciendo uso de una red de tipo crane en ProModel.

\section{MARCO CONCEPTUAL}

Al revisar fuentes bibliográficas se encuentran muchas publicaciones que utilizan la simulación discreta; entre ellas tenemos las siguientes.

Trujillo, Vallejo y Becerra (2010) utilizan la simulación para modelar un centro de llamadas con el ProModel $\circledast$, que permita identificar las pruebas de bondad de ajuste y homogeneidad con el fin de formular propuestas de mejoramiento para la productividad del sistema. Entre ellas plantearon el requerimiento de nuevo personal e identificaron cuellos de botella.

El propósito del articulo según Taddei, Rodríguez y Ruiz (2013), es mostrar la utilización de la simulación de sistemas, enfocada al sector de los servicios, para resolver problemas de asignación de recursos y toma de decisiones en el proceso de Inscripciones de Primer Ingreso en el campus Hermosillo de la Universidad de Sonora, en el norte de México, teniendo como objetivo encontrar el número óptimo de servidores requeridos en los subprocesos involucrados, sin comprometer la calidad del servicio. El artículo muestra cómo la aplicación de la simulación de sistemas permite hacer más eficiente el uso de los recursos, reducir 
los tiempos de espera y brindar mejor atención, en este caso a los aspirantes a ingresar a la Universidad de Sonora. Se aplican este tipo de simulaciones con alta frecuencia, ya que permiten modelar los procesos y las políticas de los servicios.

A través de este articulo Roldán, Moras y Aguilar (2007) se mostró la utilización de la simulación por medio de un software Ilamado Promodel $\mathbb{R}$, como una herramienta para el mejoramiento de la logística en la empresa Fricongelados Citlaltépetl en Orizaba, Veracruz, México. Se hizo un modelo representativo del sistema real de reparto del helado, y a partir de este modelo se hizo otro con las mejoras propuestas para optimizar la entrega del producto y con ello incrementar la productividad de la distribuidora. Para efectuar el análisis estadístico de los tiempos de servicio en los puntos de venta se utilizó el programa Stat:Fit que incor-

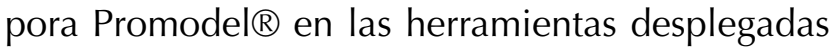
en su menú inicial. En dicho programa se aplicaron las pruebas de bondad de ajuste chi cuadrado, Kolmogorov-Smirnov y Anderson-Darling a todos los datos recolectados para determinar a qué distribución de probabilidad teórica conocida (p. ej., uniforme, exponencial, normal, etc.) se ajustaba dicha información.

Según Coss (1993) la simulación es el proceso de diseñar y desarrollar un modelo computarizado de un sistema o proceso y conducir experimentos con este modelo con el propósito de entender el comportamiento del sistema o evaluar varias estrategias con las cuales se puede operar el sistema.

ProModel es un software de simulación utilizado por empresas de todo el mundo para simular sus operaciones en la búsqueda de mejoras en la productividad, optimización de la producción, disminución de costos, etc., así como para la evaluación de ideas y diseños de nuevos sistemas. Está disponible en: http://www.simulart.cl/industrias/ universidades-y-academicos.

Al revisar la literatura se observa la diversidad de trabajos que se pueden desarrollar con el uso de la simulación. Lo anterior sirvió de referente para simular un modelo del sistema actual de la celda de manufactura flexible de la Facultad de Ingeniería Industrial de la Universidad Tecnológica de Pereira. Cabe señalar que simular no es una tarea fácil.

\section{METODOLOGÍA}

Etapas de la simulación del modelo de la FMC. Los pasos necesarios para realizar la simulación de la celda de manufactura flexible de la Facultad de Ingeniería Industrial de la Universidad Tecnológica de Pereira son los siguientes (Coss, 1993).

\section{Definición del sistema}

La Facultad de Ingeniería Industrial de la Universidad Tecnológica de Pereira cuenta con una Celda de Manufactura Flexible (FMC) que está compuesta por las siguientes estaciones de trabajo: un almacén de materia prima, un robot para descargue de material, una banda transportadora, pallets de transporte, fresadora $\mathrm{CNC}$ (con control numérico por computadora), y un brazo robot MITSUBISHI MELFA RV; este último cuenta con la posibilidad de desplazarse a través de una guía, para llevar materiales desde la banda transportadora a la fresadora CNC y viceversa. También cuenta con un software Minitek que permite el montaje y alistamiento de las estaciones de trabajo, planeación de los procesos de la ruta de producción de una pieza (ver la figura 1).

\section{Formulación del modelo}

Una vez definido con exactitud el proceso que se efectúa en la celda de manufactura flexible, se pasa a definir las variables y flujograma que describe en forma compleja al modelo.

\section{Definición de variables}

Número de piezas en proceso, número de piezas fresadas, número de piezas terminadas, piezas procesadas en el almacén y tiempo de ciclo. 


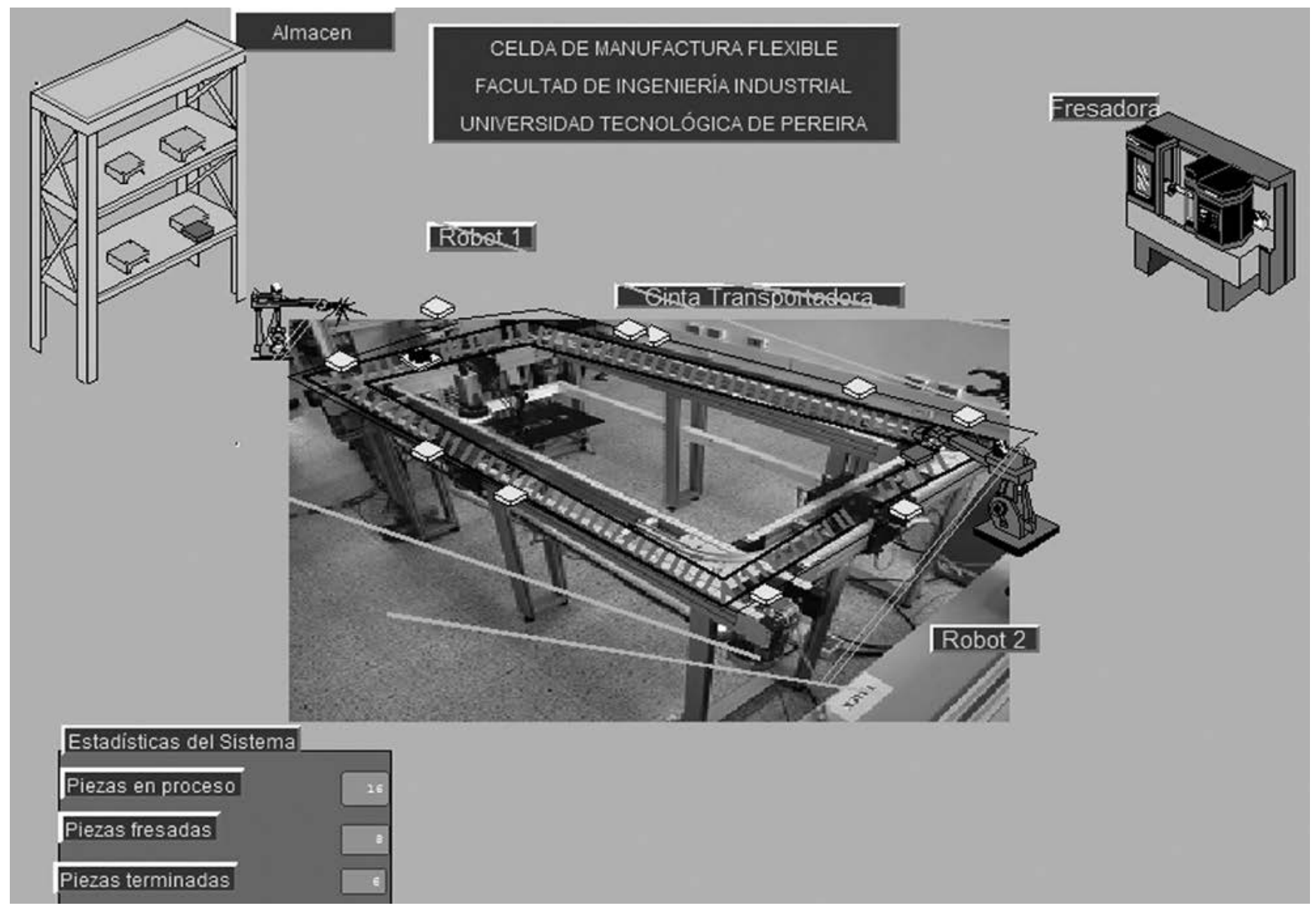

Figura 1. Layout del modelo en Promodel.

Fuente: ProModel.

\section{Flujograma del modelo}

En la figura 2 se puede observar los pasos de cada proceso del modelo.

El proceso que se realiza en la FMC que se refleja en la figura 2 es el siguiente: Se ubica una pieza prismática en una de las posiciones del almacén, después de esto se manipula el software para que efectúe las siguientes tareas:

El robot que está situado entre el almacén y la cinta transportadora debe coger la pieza prismática y ponerla en un palet de la cinta transportadora; la cinta trasportadora inicia su movimiento y transporta la pieza hasta la ubicación del robot
MITSUBISHI MELFA que está del otro lado del almacén y localizado entre un extremo de la cinta transportadora. El robot coge la pieza prismática y la lleva hacia la fresadora, la fresadora hace todo un proceso en donde inicia con abrir la puerta para que el robot ubique la pieza y espera a que el robot se retire e inicia con el proceso que está asociado al programa que le indican en el sistema que debe realizar a la pieza; por ejemplo, un programa que dibuje en la pieza prismática un corte que simule el diseño de una tapa. Cuando termina de correr todas las instrucciones del software, automáticamente se detiene y se abre la puerta para que el robot nuevamente tome la pieza procesada 


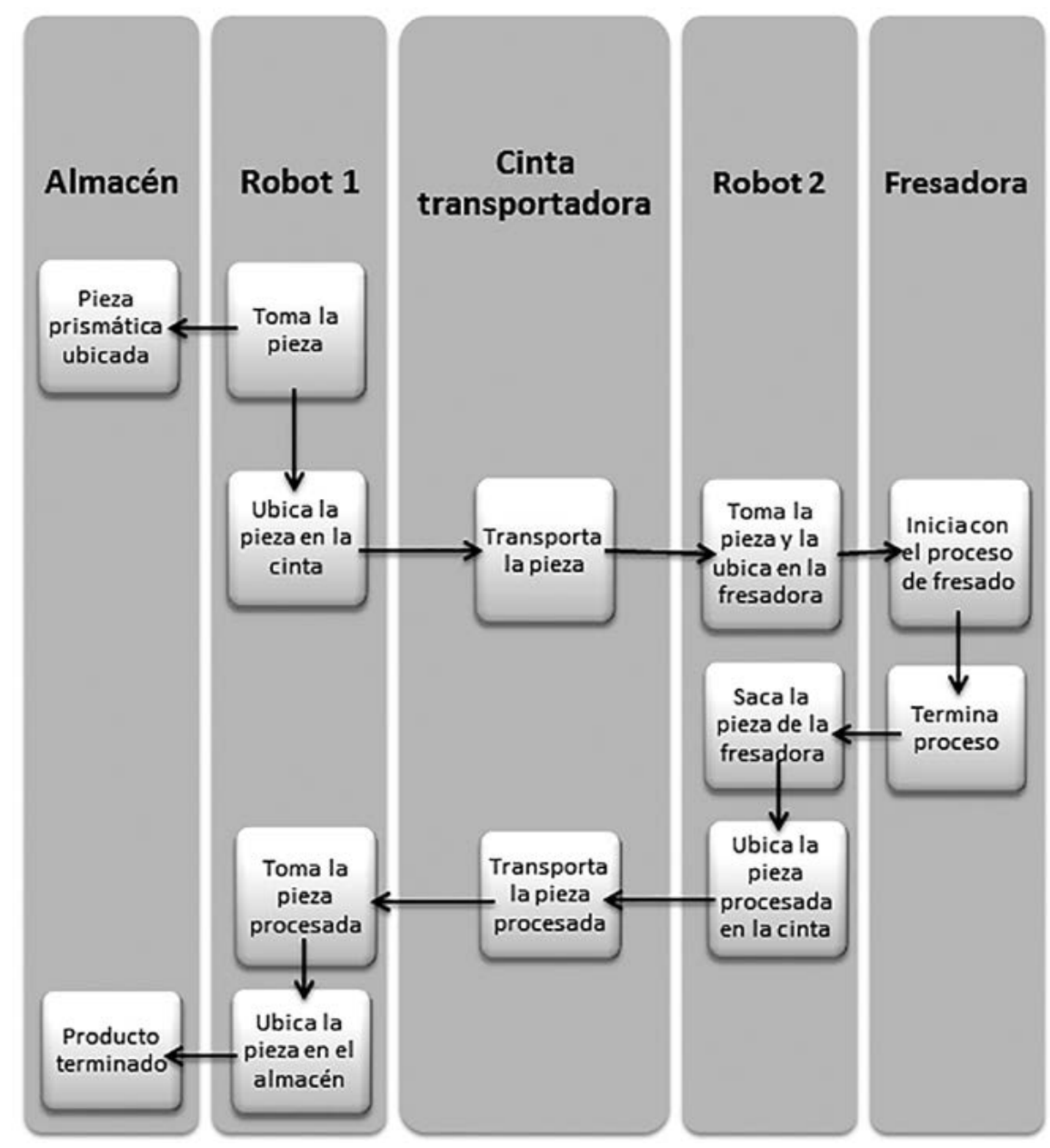

Figura 2. Flujograma del modelo.

Fuente: elaboración propia.

y la lleve al palet que está en la cinta transportadora. La cinta transportadora inicia su movimiento y lleva la pieza procesada al otro extremo en donde está el almacén, la cinta para y actúa el robot llevando la pieza procesada a su lugar en el almacén como producto terminado.

\section{Recolección de datos}

El Minitek, software que controla la celda de manufactura flexible tiene una opción de registros históricos en donde se puede visualizar información de las planificaciones que se han lanzado a producción y la lista de órdenes de fabricación ya producidas, mostrando información como total y mínimo tiempo de fabricación. También se puede observar un Gantt de la producción.

El Promodel permite visualizar los resultados de la simulación por medio de tablas y un visualizador para generar gráficos como histogramas, entre otros.

Teniendo en cuenta lo anterior es posible obtener datos que pueden describir el comportamiento de los procesos con el fin de mejorar la productividad. 


\section{Implementación}

La simulación de estaciones de trabajo de una celda de manufactura flexible comienza por el reconocimiento del sistema e identificar la interacción existente que se requiere para hacer un modelo proactivo. Dicho modelo se desarrolla en "ProModel, el cual está diseñado para modelamiento de sistemas de fabricación que van desde pequeños talleres de trabajo y las células de mecanizado a gran escala de producción y los sistemas de fabricación flexibles" (Harrell, 2013). Los elementos básicos de modelado en ProModel son: locaciones, entidades, llegadas y procesos (Harrell, 2004). Las locaciones representan lugares fijos en el sistema; cualquier cosa que un modelo puede procesar se llama una entidad. En la figura 3 se encuentra la descripción de las locaciones y de las entidades.
Algunos ejemplos son partes de una fábrica, los pacientes de un hospital, los clientes de un banco y los viajeros que llaman para hacer reservaciones aéreas. El mecanismo para definir cómo las entidades entran en el sistema se llama llegadas, las cuales se encuentran detalladas en la figura 6. Las entidades pueden llegar individualmente o en lotes. El tiempo entre las llegadas de entidades sucesivas se llama tiempo entre llegadas. El procesamiento describe las operaciones que tienen lugar en una locación, como la cantidad de tiempo que una entidad pasa allí, los recursos que necesita para completar el proceso, y cualquier cosa que sucede en el lugar, ProModel también incluye otros elementos, como la red de caminos descrita en la figura 4, atributos, recursos descritos en la figura 4, variables detalladas en la figura 6, los nodos asociados a los recursos referidos en la figura

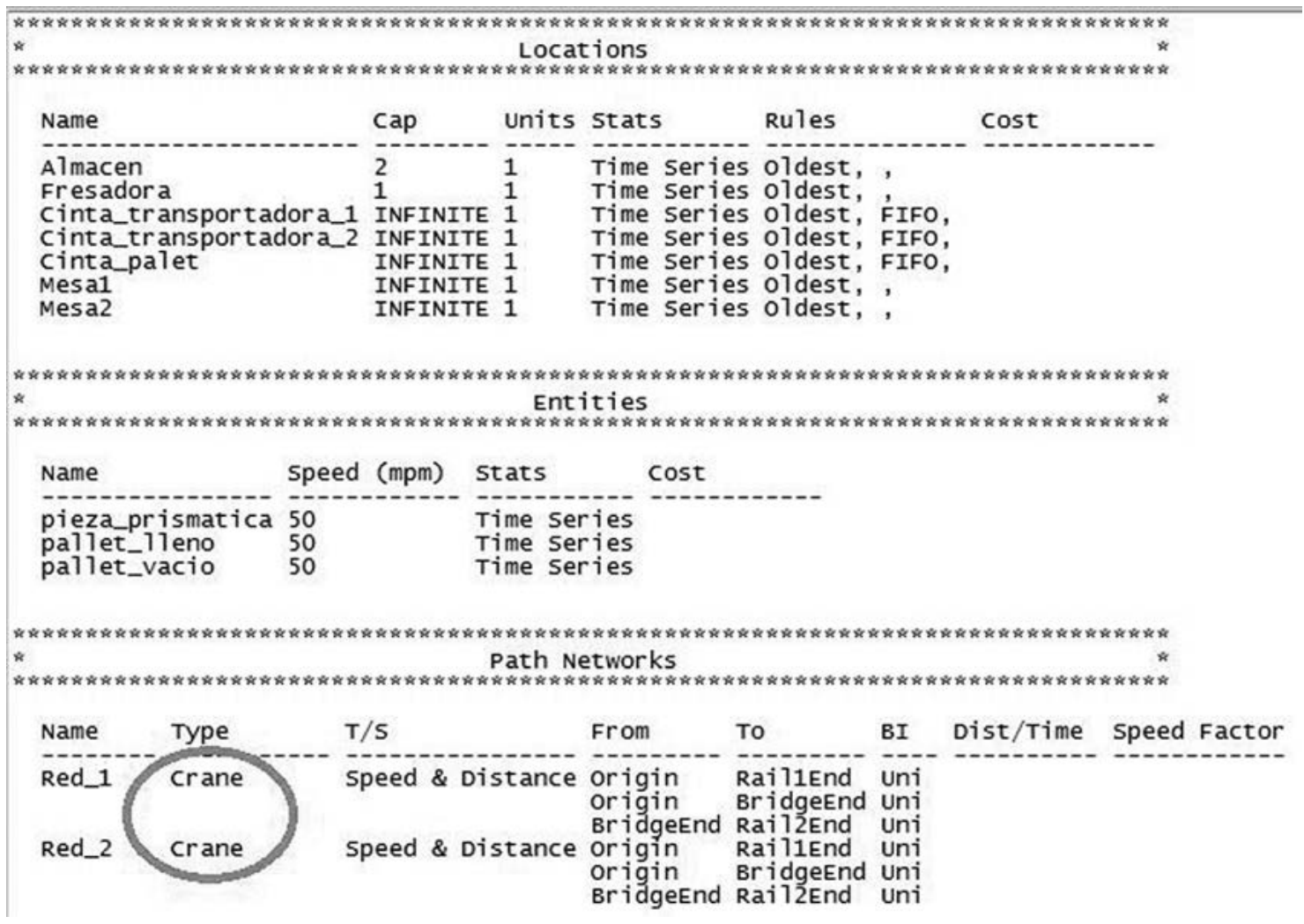

Figura 3. Modelado del sistema de una celda de manufactura flexible en Promodel.

Fuente: ProModel. 
5, etc. ProModel se centra en la utilización de recursos, la producción, la capacidad, la productividad, los niveles de inventario, cuellos de botella, tiempos de producción y otras medidas de rendimiento. ProModel es capaz de modelar los más complejos sistemas (Yu, 2006). La descripción del proceso se encuentra en la tabla 1.

Crane: es un sistema de grúas que forma una zona representado en paralelogramo, delimitada por dos carriles y las líneas que conectan los puntos finales de los rieles. Las líneas que conectan los puntos extremos de los carriles son efectivamente las dos posiciones extremas de las líneas centrales de los puentes que operan dentro de la trayectoria. ProModel utiliza el final de uno de los carriles como el origen para servir como punto de referencia para todos los cálculos de distancia dentro de la trayectoria.

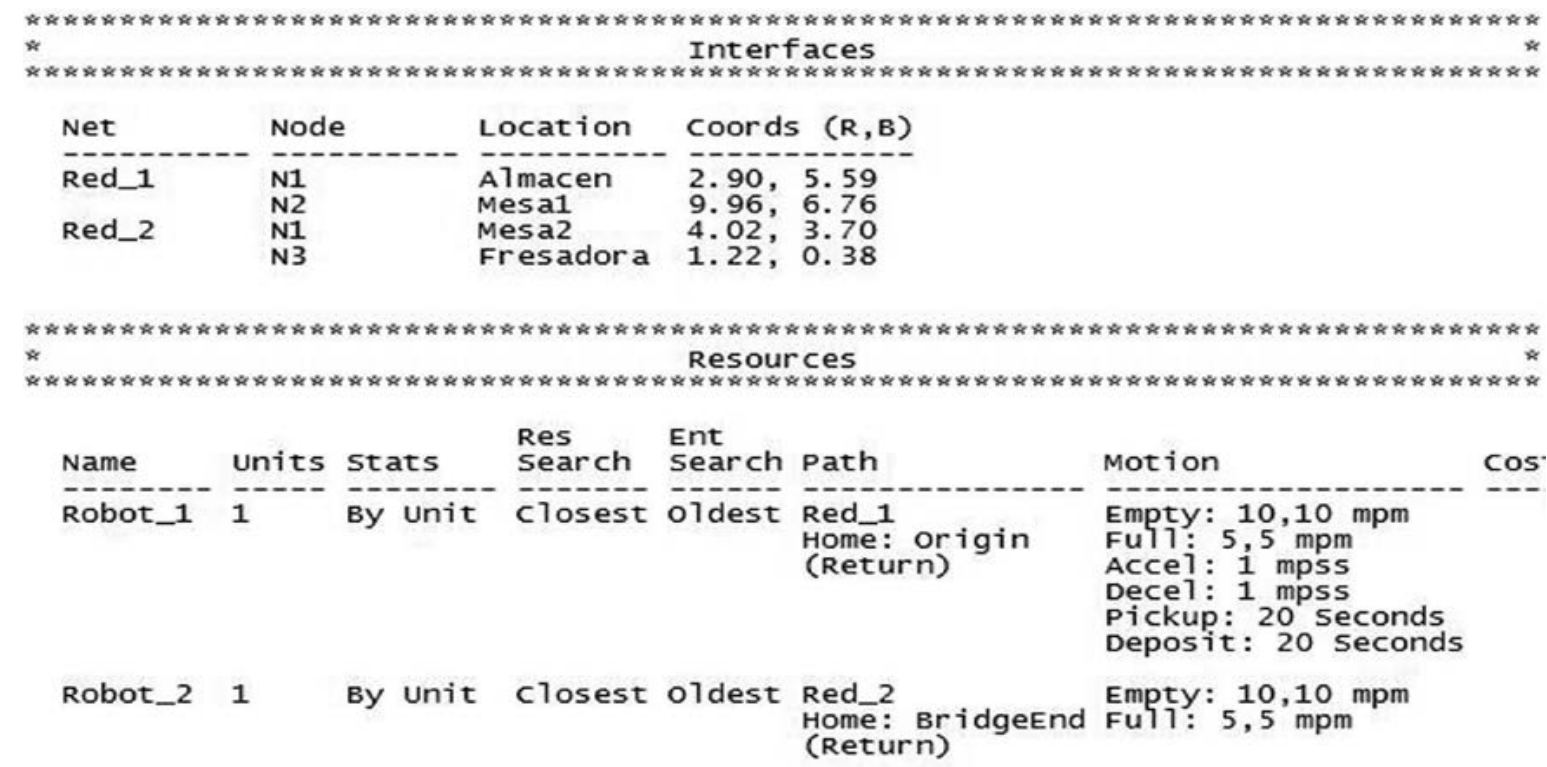

Figura 4. Descripción recursos y redes.

Fuente: Promodel.

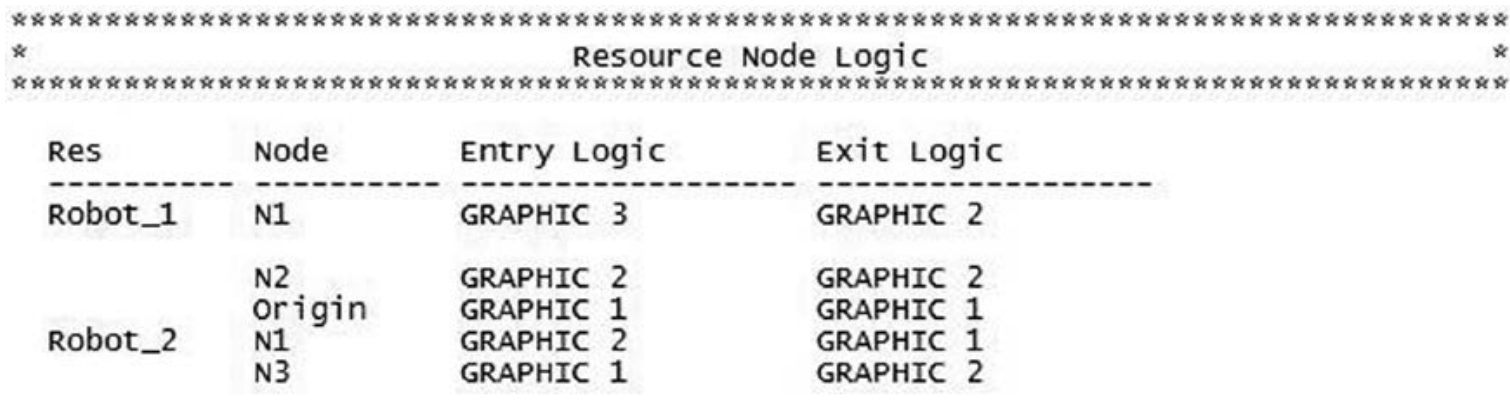

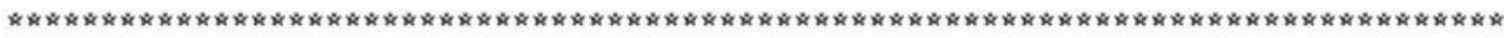

Figura 5. Nodos asociados a los recursos.

Fuente: Promodel. 
Tabla 1. Descripción del proceso.

\begin{tabular}{|c|c|c|c|c|c|c|c|}
\hline \multicolumn{8}{|c|}{ Processing } \\
\hline \multicolumn{5}{|c|}{ Process } & \multicolumn{2}{|c|}{ Routing } & \multirow[b]{2}{*}{ Move Logic } \\
\hline Entity & Location & Operation & Blk & Output & Destination & Rule & \\
\hline $\begin{array}{l}\text { pieza_ } \\
\text { prismática }\end{array}$ & Almacén & $\begin{array}{l}\text { Tiempo_entrada= } \\
\text { CLOCK(MIN) WAIT } 1\end{array}$ & 1 & $\begin{array}{l}\text { pieza_ } \\
\text { prismática }\end{array}$ & Mesa1 & LOAD 1 & $\begin{array}{l}\text { INC Piezas_en_- } \\
\text { proceso MOVE } \\
\text { WITH Robot_1 } \\
\text { THEN FREE }\end{array}$ \\
\hline pallet_vacío & Cinta_palet & & 1 & pallet_vacío & Mesa1 & FIRST 1 & \\
\hline pallet_vacío & Mesa1 & $\begin{array}{l}\text { LOAD } 1 \\
\text { WAIT } 0.16\end{array}$ & 1 & pallet_Ileno & $\begin{array}{l}\text { Cinta_- } \\
\text { transportado- } \\
\text { ra_1 }\end{array}$ & FIRST 1 & \\
\hline pallet_lleno & $\begin{array}{l}\text { Cinta_transporta- } \\
\text { dora_1 }\end{array}$ & & 1 & pallet_Ileno & Mesa2 & FIRST 1 & \\
\hline pallet_lleno & Mesa2 & $\begin{array}{l}\text { UNLOAD } 1 \\
\text { WAIT } 1\end{array}$ & 1 & pallet_vacío & Cinta_palet & FIRST 1 & \\
\hline $\begin{array}{l}\text { pieza__ } \\
\text { prismática }\end{array}$ & Mesa2 & & 1 & $\begin{array}{l}\text { pieza_ } \\
\text { prismática }\end{array}$ & Fresadora & FIRST 1 & $\begin{array}{l}\text { MOVE WITH Ro- } \\
\text { bot_2 THEN FREE }\end{array}$ \\
\hline $\begin{array}{l}\text { pieza__ } \\
\text { prismática }\end{array}$ & Fresadora & $\begin{array}{l}\text { tiempo_proceso_fre- } \\
\text { sadora= } \\
\text { CLOCK(MIN) WAIT } 5\end{array}$ & 1 & $\begin{array}{l}\text { pieza_ }_{\text {prismática }}\end{array}$ & Mesa2 & FIRST 1 & $\begin{array}{l}\text { GRAPHIC } 2 \text { LOG } \\
\text { "Tiempo de proce- } \\
\text { so en fresadora", } \\
\text { tiempo_proceso_ } \\
\text { fresadora } \\
\text { MOVE WITH Ro- } \\
\text { bot_2 THEN FREE } \\
\text { INC Piezas_fre- } \\
\text { sadas }\end{array}$ \\
\hline pallet_vacío & Cinta_palet & & 1 & pallet_vacío & Mesa2 & FIRST 1 & \\
\hline pallet_vacío & Mesa2 & $\begin{array}{l}\text { LOAD } 1 \\
\text { WAIT } 1\end{array}$ & 1 & pallet_lleno & $\begin{array}{l}\text { Cinta_- } \\
\text { transportado- } \\
\text { ra_2 }\end{array}$ & FIRST 1 & \\
\hline pallet_lleno & $\begin{array}{l}\text { Cinta_transporta- } \\
\text { dora_2 }\end{array}$ & & 1 & pallet_lleno & Mesa1 & FIRST 1 & \\
\hline pallet_lleno & Mesa1 & $\begin{array}{l}\text { UNLOAD } 1 \\
\text { WAIT } 2\end{array}$ & 1 & pallet_vacío & cinta_pallet & FIRST 1 & $\begin{array}{l}\text { GRAPHIC } 2 \\
\text { MOVE WITH Ro- } \\
\text { bot_1 THEN FREE } \\
\text { INC Piezas_proce- } \\
\text { sadas_almacén }\end{array}$ \\
\hline $\begin{array}{l}\text { pieza__ } \\
\text { prismática }\end{array}$ & Mesa1 & $\begin{array}{l}\text { GRAPHIC } 2 \\
\text { WAIT } 1\end{array}$ & 1 & $\begin{array}{l}\text { pieza_ } \\
\text { prismática }\end{array}$ & Almacén & FIRST 1 & \\
\hline $\begin{array}{l}\text { pieza__ } \\
\text { prismática }\end{array}$ & Almacén & $\begin{array}{l}\text { LOG “Tiempo de Ci- } \\
\text { clo", tiempo_entrada }\end{array}$ & 1 & $\begin{array}{l}\text { pieza_ } \\
\text { prismática }\end{array}$ & EXIT & FIRST 1 & $\begin{array}{l}\text { INC Piezas_termi- } \\
\text { nadas } \\
\text { DEC Piezas_en_- } \\
\text { proceso }\end{array}$ \\
\hline
\end{tabular}

Fuente: elaboración propia. 


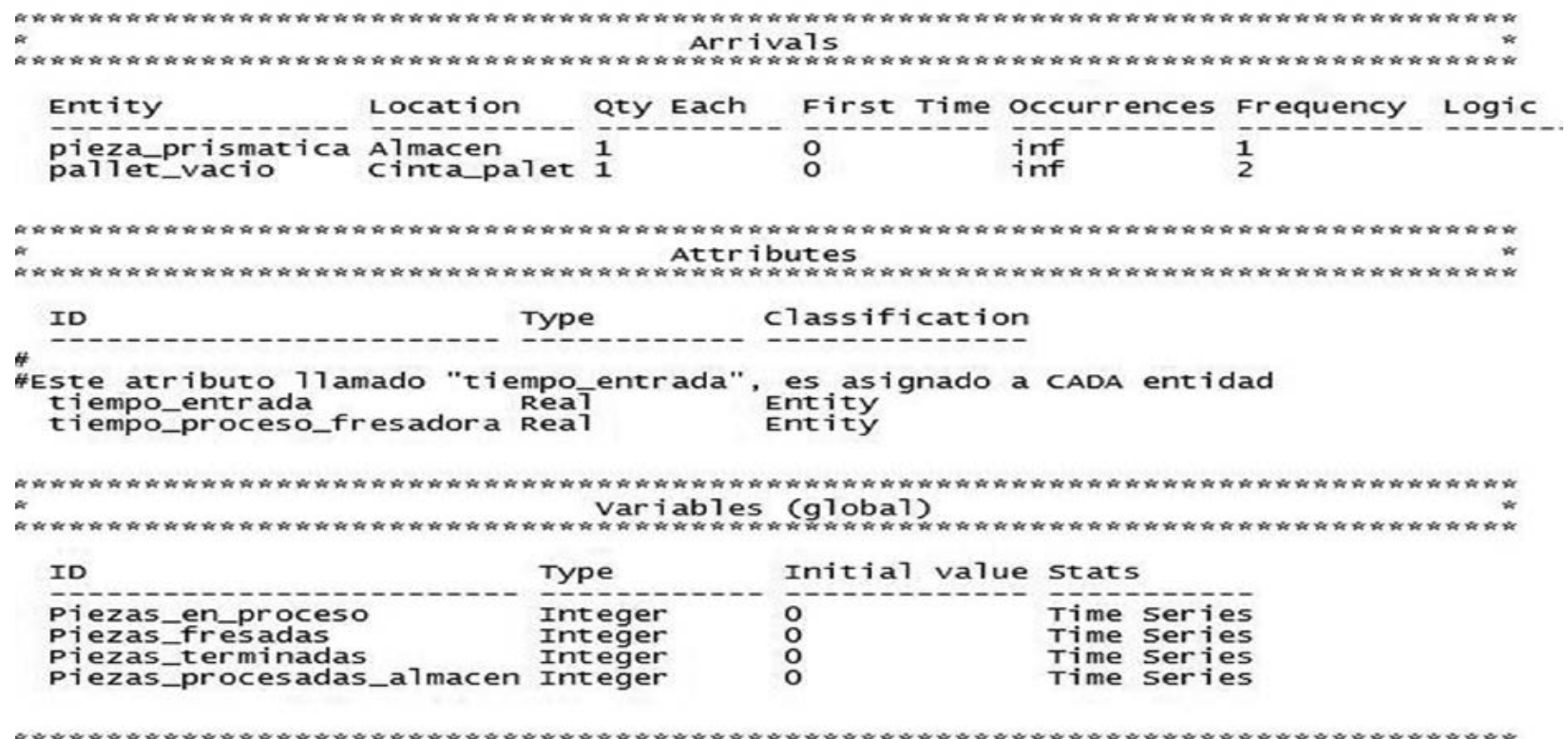

Figura 6. Descripción de llegadas, atributos y variables.

Fuente: ProModel.

\section{RESULTADOS}

Para analizar el comportamiento de los procesos con el fin de mejorar la productividad de la simulación de la celda de manufactura flexible se tuvo en cuenta los resultados obtenidos, los cuales se muestran en la figura 7 y tabla 2.

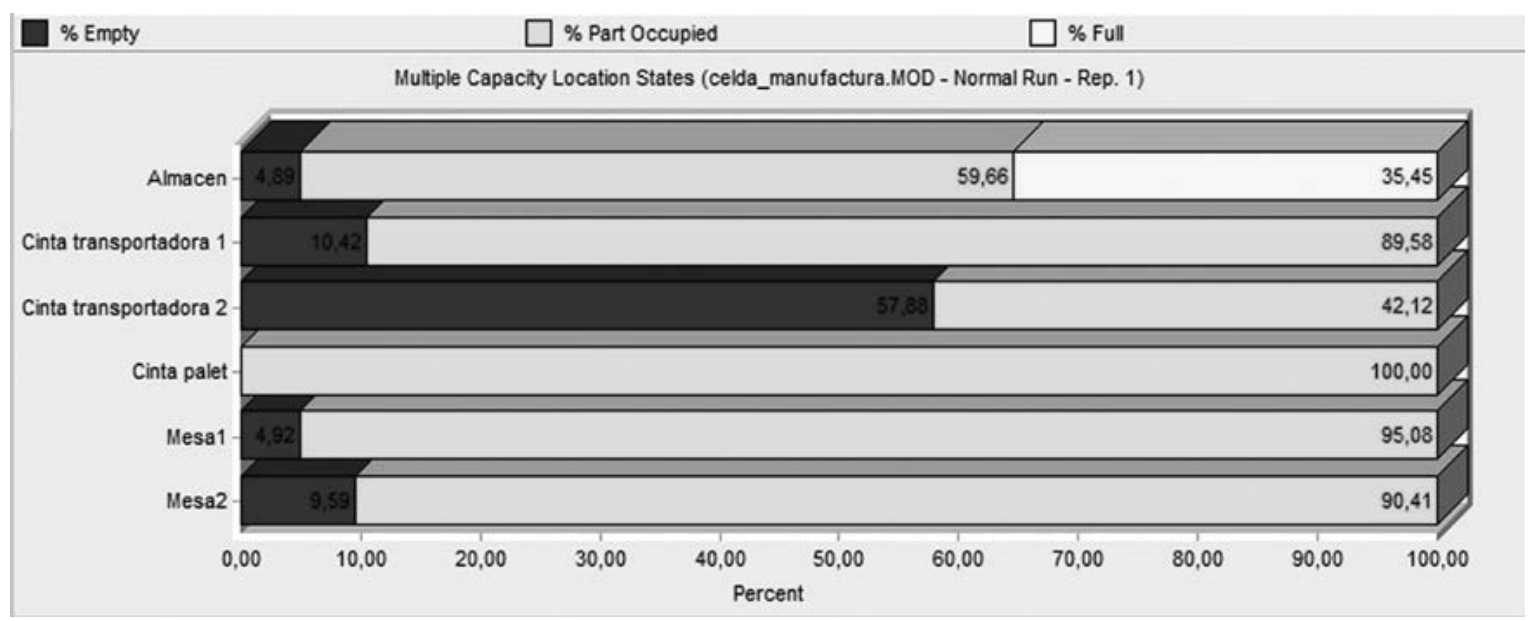

Figura 7. Capacidad de las locaciones.

Fuente: ProModel. 
Tabla 2. Descripción del proceso.

\begin{tabular}{cccccccc}
\hline Name & $\begin{array}{c}\text { Scheduled } \\
\text { Time (HR) }\end{array}$ & \% Operation & \% Setup & \% Idle & \% Waiting & \% Blocked & \% Down \\
\hline Fresadora & 3 & 63,89 & 0 & 25,42 & 10,69 & 0 & 0 \\
\hline
\end{tabular}

Fuente: ProModel.

Se puede observar en la tabla 3 y tabla 4 que la locación almacén está ocupada en $59,6 \%$, vacía en $4,89 \%$ y llena completamente en $35,45 \%$. La cinta transportadora 2 presenta un alto grado de vacío $(57,88 \%)$; lo anterior se puede presentar por el proceso que debe realizar la fresadora y hasta que se termine dicho proceso el robot 2 no puede entregar a la cinta transportadora 2 el material para continuar con el proceso. Estos resultados son bastante eficientes ya que podemos observar altos porcentajes de ocupación de las locaciones, exceptuando las locaciones de almacén y cinta transportadora 2, que se deben estudiar a fondo para ver qué sucede y poder aumentar la utilización de las mismas. La locación fresadora no presenta bloqueos, punto que también aumenta la productividad.

Tabla 3. Locaciones del modelo.

\begin{tabular}{|c|c|c|c|c|c|c|c|c|}
\hline \multicolumn{9}{|c|}{ celda_manufactura.MOD (Normal Run-Rep. 1) } \\
\hline Name & $\begin{array}{l}\text { Scheduled } \\
\text { Time (HR) }\end{array}$ & Capacity & $\begin{array}{c}\text { Total } \\
\text { Entries }\end{array}$ & $\begin{array}{l}\text { Avg Time Per } \\
\text { Entry (MIN) }\end{array}$ & $\begin{array}{c}\text { Avg } \\
\text { Contents }\end{array}$ & $\begin{array}{c}\text { Maximum } \\
\text { Contents }\end{array}$ & $\begin{array}{l}\text { Current } \\
\text { Contents }\end{array}$ & $\stackrel{\%}{\text { Utilization }}$ \\
\hline Almacén & 3 & 2,00 & 73 & 3,22 & 1,31 & 2 & 1 & 65,28 \\
\hline Fresadora & 3 & 1,00 & 23 & 5,84 & 0,75 & 1 & 1 & 74,58 \\
\hline Cinta transportadora 1 & 3 & 999999,00 & 51 & 3,75 & 1,06 & 2 & 1 & 2,14 \\
\hline Cinta transportadora 2 & 3 & 999999,00 & 22 & 3,45 & 0,42 & 1 & 0 & 0,94 \\
\hline Cinta palet & 3 & 999999,00 & 159 & 8,63 & 7,63 & 10 & 8 & 2,45 \\
\hline Mesa1 & 3 & 999999,00 & 126 & 34,40 & 24,08 & 55 & 54 & 0,00 \\
\hline Mesa2 & 3 & 999999,00 & 97 & 44,79 & 24,14 & 52 & 52 & 0,00 \\
\hline
\end{tabular}

Fuente: ProModel.

Se simula el modelo durante 3 horas del proceso de la celda de manufactura flexible y se obtiene un inventario final de 73 unidades que llegaron al almacén. Hay que tener en cuenta que en estas 73 piezas hay piezas sin fresar y piezas fresadas, que son los productos terminados. Lo anterior porque el almacén recibe la materia prima y el producto final; para lograr un mejor análisis se debe recurrir al resultado de las variables. La locación que tiene mayor porcentaje de utilización es la fresadora, ese alto porcentaje podría estar relacionado con el tiempo de proceso de la locación. A continuación, en la tabla 4, podemos observar el resultado de las variables. 
Tabla 4. Variables del modelo.

\begin{tabular}{lc}
\hline \multicolumn{1}{c}{ Name } & Current Value \\
\hline Piezas en proceso & 31 \\
\hline Piezas fresadas & 22 \\
\hline Piezas terminadas & 21 \\
\hline Piezas procesadas almacén & 21 \\
\hline
\end{tabular}

Fuente: elaboración propia

Se define la productividad en un puesto de trabajo como la relación existente entre un conjunto de unidades procesadas por unidad de tiempo (Sempere, 2008), obteniendo la siguiente ecuación:

$$
\text { Productividad }=\frac{\text { unidades procesadas }}{\text { tiempo }}
$$

Teniendo en cuenta la ecuación (1), para medir la productividad de los resultados del modelo, con miras al mejoramiento para la productividad del sistema se tuvo en cuenta la siguiente medida de desempeño:

Calcular productividad $=23 / 3=7$ piezas procesadas por hora

Este valor se constituye en uno de los principales indicadores, ya que establece el rendimiento del modelo en una corrida de simulación.

Tabla 5. Recursos del modelo.

\begin{tabular}{ccccccccc}
\hline \multicolumn{7}{c}{ celda_manufactura.MOD (Normal Run-Rep. 1) } \\
\hline Name & Units & $\begin{array}{c}\text { Scheduled } \\
\text { Time (HR) }\end{array}$ & $\begin{array}{c}\text { Number } \\
\text { Times Used }\end{array}$ & $\begin{array}{c}\text { Avg Time } \\
\text { Per Usage } \\
\text { (MIN) }\end{array}$ & $\begin{array}{c}\text { Avg Time } \\
\text { Travel To } \\
\text { Use (MIN) }\end{array}$ & $\begin{array}{c}\text { Avg Time } \\
\text { Travel To } \\
\text { Park (MIN) }\end{array}$ & $\begin{array}{c}\text { \% Blocked } \\
\text { In Travel }\end{array}$ & $\begin{array}{c}\text { \% } \\
\text { Utilization }\end{array}$ \\
\hline Robot 1 & 1,00 & 3,00 & 72,00 & 2,08 & 0,29 & 0,00 & 0,00 & 95,08 \\
\hline Robot 2 & 1,00 & 3,00 & 45,00 & 0,66 & 0,34 & 0,68 & 0,00 & 25,41 \\
\hline
\end{tabular}

Fuente: Promodel.

El número de veces que se utiliza cada uno de los robots en cada una de las secciones es 72 y 45 para el robot 1 y robot 2 , respectivamente. El tiempo promedio de cada robot en cada una de las secciones fue 2,08 y 0,66 minutos. El recurso más utilizado es el robot 1 . Lo anterior se puede observar en la figura 9, donde se detalla los resultados de la operación de los recursos en el modelo.

\section{CONCLUSIONES}

La metodología utilizada en este trabajo permitió evaluar la productividad de un proceso que se lleva a cabo en una celda de manufactura flexible, por medio del Promodel que permite la modelación y análisis para la mejora de procesos. Este tipo de metodología puede extenderse para la evaluación de otros sistemas como manejo de materiales, prestación de servicios, entre otros. Sin embargo, la mayor dificultad para modelar se centra en comprender muy bien el sistema actual.

La simulación del modelo permitió construir y validar una metodología para el mejoramiento de procesos y maximización del uso de los recursos, dado que la experimentación sobre el modelo ayuda a comprender el comportamiento del sistema y a la toma de decisiones, las cuales pueden aumentar la productividad con un mejor desempeño del sistema (Soto, 2010).

La utilización de las etapas de la simulación como definición del sistema, formulación del modelo, identificación de variables, recolección de 
datos, implementación e interpretación, permiten una mayor comprensión de la realidad del sistema que se está simulando.

\section{FINANCIAMIENTO}

El artículo es uno de los resultados del proyecto de investigación "Metodología para el mejoramiento de procesos a través de la ingeniería de métodos y la manufactura esbelta en busca de la maximización de la utilización de los recursos-segunda etapa", radicado en la Vicerrectoría de Investigaciones Innovación y extensión de la Universidad Tecnológica de Pereira con el código 7-12-1. El proyecto se encuentra en la categoría sin financiación.

\section{REFERENCIAS}

Coss, R. (1993). Simulación un enfoque práctico. México D.F.: Limusa.

Harrell, C. y Price R. (2003). Simulation modeling using ProModel technology. Proceedings of the 2003 Winter Simulation Conference.

Harrell, C.; Ghosh, B. y Bowden, R. (2004). Simulation Using ProModel. (2a. ed.) Mc Graw-Hill.

Kale, N. ; Zottolo, M. ; Ulgen, O. y Williams E. (2007). Simulation improves end-of-line sortation and material handling pickup scheduling at appliance manufacturer. Winter Simulation Conference-WSC.
Roldán, E.; Moras, C., y Aguilar, A. (2007). Optimización de las rutas de reparto de helado de la empresa Fricongelados Citlaltépetl. Ingeniería Industrial, Vol. 1, No. 1. Disponible en: http://academiajournals.com/downloads/RoldanMorasAquilar.pdf [Consulta: 2014-05-14].

Sampere, F.; Miralles, C.; Romano, C. y Vincens, E. (2008). Aplicación de mejora de métodos de trabajo y medición de tiempos. México D.F.: Limusa.

Soto, J. (2010). Laboratorios de simulación discreta. Colombia: Postergraph S.A.

Taddei, J.; Rodríguez, R., y Ruiz, J. (2013). Mejora del proceso de inscripciones en una Institución de Educación Superior mediante Simulación. Ingeniería Industrial, vol. 34, No. Disponible en: http://scielo.sld.cu/scielo.php?pi$d=S 1815-59362013000100003 \&$ script=sci_arttext. [Consulta: 2013-05-10].

Trujillo, J.; Vallejo, J., y Becerra, M. (2010). Metodología para la simulación de centros de llamadas-Caso de estudio. Studiositas, vol. 5, No. 3, pp. 117-137. Disponible en: http://dialnet.unirioja.es/servlet/articulo? codigo=3951276. [Consulta: 2014-05-12].

Yu, Q.; Duffy, V. y Zachary, J. (2006). Productivity simulation with Promodel for an automotive assembly workstation involving a lift assist device. Proceedings of the 2006 Winter Simulation Conference.

\section{(C) $(1) \Theta$}

